\title{
Biowaste Lignin-Based Carbonaceous Materials as Anodes for Na-Ion Batteries
}

\author{
Cyril Marino, $\oplus^{\mathrm{z}}$ Joel Cabanero, Mauro Povia, and Claire Villevieille \\ Paul Scherrer Institut, Electrochemistry Laboratory, CH-5232 Villigen PSI, Switzerland
}

\begin{abstract}
Carbonaceous materials derived from biomass lignin-based precursors are an attractive alternative to the hard carbon materials generally used in Na-ion batteries. In this work, we employed almond shells as biowaste precursors and investigated the impact of the annealing atmosphere $\left(\mathrm{Ar}, \mathrm{N}_{2}\right.$, or $\left.\mathrm{Ar} / \mathrm{H}_{2}\right)$ on the physicochemical and the electrochemical properties of the obtained carbonaceous materials. Raman spectroscopy, Brunauer-Emmett-Teller analysis, and scanning electron microscopy indicated a relationship between the porosity and the annealing atmosphere. Under a reductive atmosphere, the surface chemistry of the sample was modified, which had an impact on the electrochemical performance. The materials synthesized under Ar and $\mathrm{N}_{2}$ atmospheres delivered specific charges of ca. $255 \mathrm{mAh} \cdot \mathrm{g}^{-1}$, which were sustained for more than 60 cycles, whereas the electrochemical performance of the carbonaceous material synthesized under a reductive atmosphere $\left(\mathrm{Ar} / \mathrm{H}_{2}\right)$ was drastically diminished. Once the optimal synthesis conditions were determined, other lignin-derived biowaste materials, such as walnut shells and scrap wood, were also investigated. Despite having similar physicochemical properties, the carbonaceous material derived from scrap wood exhibited better electrochemical performance (specific charge of $270 \mathrm{mAh} \cdot \mathrm{g}^{-1}$ ), confirming the impact of morphology on the electrochemical performance.

(C) The Author(s) 2018. Published by ECS. This is an open access article distributed under the terms of the Creative Commons Attribution 4.0 License (CC BY, http://creativecommons.org/licenses/by/4.0/), which permits unrestricted reuse of the work in any medium, provided the original work is properly cited. [DOI: $10.1149 / 2.0681807 \mathrm{jes}]$

(cc) BY
\end{abstract}

Manuscript submitted March 27, 2018; revised manuscript received April 18, 2018. Published May 9, 2018.

Despite the efforts made toward waste reduction, the amount of trash is expected to double by $2025 .{ }^{1}$ Trash generally contains some compost and biowaste materials, and recycling such materials could lead to the development of a primary source that can be used subsequently for electrode materials. Recently, Na-ion batteries have been proposed as a complementary system to lithium technology, but these batteries will only be an alternative to Li-ion batteries in certain niche applications unless the development costs are decreased. Thus, using biowaste materials as electrode materials could be a logical way of decreasing the electrode material cost.

With this objective, Schröder et al. ${ }^{2}$ synthesized activated carbons from biomass waste, such as rice straw, olive stones, wheat straw, and walnut shells. They demonstrated that the synthesis conditions could be used to tune and adapt the physical properties of the carbons for various applications. Different carbon precursors, such as fruits, sugars, natural polymers, and proteins, have been reported. ${ }^{3-5} \mathrm{Wu}$ et al. ${ }^{6}$ used apple waste treated at $1100^{\circ} \mathrm{C}$ for $6 \mathrm{~h}$ under $\mathrm{Ar}$ and obtained a stable specific charge of $245 \mathrm{mAh} \cdot \mathrm{g}^{-1}$ for 80 cycles at a rate of $0.1 \mathrm{C}$ (considering $1 \mathrm{C}=200 \mathrm{~mA} \cdot \mathrm{g}^{-1}$ and an electrode loading of $\left.1 \mathrm{mg} \cdot \mathrm{cm}^{-2}\right)$. Moreover, at a higher current density $\left(>10 \mathrm{~mA} \cdot \mathrm{g}^{-1}\right)$, pomelo peels carbonized at $700^{\circ} \mathrm{C}$ for $2 \mathrm{~h}$ under $\mathrm{N}_{2}$ delivered a reversible specific charge of $200 \mathrm{mAh} \cdot \mathrm{g}^{-1}$ for 200 cycles, ${ }^{7}$ whereas leaves treated at $1000^{\circ} \mathrm{C}$ for $1 \mathrm{~h}$ under Ar and cycled without a binder or carbon additive reached a specific charge of ca. $300 \mathrm{mAh} \cdot \mathrm{g}^{-1}$ for 60 cycles. ${ }^{8}$ However, as no information on electrode loading was available, there is some uncertainty associated with these results.

Particular attention has been given to lignin-based precursors 9 because these highly cross-linked and noncrystalline biopolymers can be used to produce non-graphitic carbons at temperatures below $1000-1100^{\circ} \mathrm{C}^{3}{ }^{3}$ Dou et al. ${ }^{4}$ compared the carbonaceous materials produced from peanut shells (lignin based) heated at $1100^{\circ} \mathrm{C}$ for $1 \mathrm{~h}$ under Ar with those produced from apple waste (pectin based) and corncob (hemicellulose based). They obtained the highest electrochemical performance, corresponding to a specific charge of $285 \mathrm{mAh} \cdot \mathrm{g}^{-1}$ sustained for 300 cycles at $0.1 \mathrm{C}$ (considering $1 \mathrm{C}=200 \mathrm{~mA} \cdot \mathrm{g}^{-1}$ and an electrode loading of $\left.1.5 \mathrm{mg} \cdot \mathrm{cm}^{-2}\right)$, for the lignin-based carbonaceous material. These results were further confirmed by an investigation of the effect of heating temperature on the carbonaceous materials obtained from argan shells. ${ }^{10}$ The sample produced at $1200^{\circ} \mathrm{C}(1 \mathrm{~h}$ treatment under $\mathrm{Ar}$ ) sustained a specific charge of $290 \mathrm{mAh} \cdot \mathrm{g}^{-1}$ for 60 cycles at $0.1 \mathrm{C}$ (considering $1 \mathrm{C}=300 \mathrm{~mA} \cdot \mathrm{g}^{-1}$ and an electrode loading of $1.5 \mathrm{mg} \cdot \mathrm{cm}^{-2}$ ). However, based on the literature, it is difficult to compare the impact of different lignin-based sources on the electrochemical performance because different synthesis procedures and electrodes have been used. Furthermore, the impact of the calcination atmosphere used during synthesis on the electrochemical results has not yet been investigated.

As both the gas atmosphere and carbon source will affect the electrochemical performance and the production cost, in this work, we decided to investigate three different lignin-based precursors: almond shells (ALM), walnut shells (WN), and scrap wood (FIR). First, we developed a synthesis protocol using ALM as a benchmark. Several atmospheres were investigated ( $\mathrm{Ar}, \mathrm{N}_{2}$, and $\left.\mathrm{Ar} / \mathrm{H}_{2}\right)$ and the impact on the electrochemical performance was assessed. Second, the synthesis protocol was applied to other lignin-based sources. All the developed carbonaceous materials were characterized by X-ray diffraction (XRD), scanning electron microscopy (SEM), BrunauerEmmett-Teller (BET) specific surface area measurements, Raman spectroscopy, and small-angle X-ray scattering (SAXS). The electrochemical performance of the developed materials was analyzed as a function of the carbon source and the gas atmosphere.

\section{Experimental}

Synthesis.-Three different carbon precursors were used: ALM, WN, and FIR (Obise SA, Switzerland). The synthesis protocol is described in Figure 1. In the first step, the raw material was ball milled for $45 \mathrm{~min}$ (active) in a $45 \mathrm{~mL}$ stainless steel vessel with three stainless steel balls (diameter: $15 \mathrm{~mm}$ ). The powder was then sieved to select particles with sizes smaller than $125 \mu \mathrm{m}$. The resulting fine yellowish powder was washed for $12 \mathrm{~h}$ in a $1 \mathrm{M} \mathrm{H}_{2} \mathrm{SO}_{4}$ solution to remove traces of pesticides, rinsed with distilled water until a neutral $\mathrm{pH}$ was reached, and dried at $80^{\circ} \mathrm{C}$ for $12 \mathrm{~h}$. Thermal treatment at $1000^{\circ} \mathrm{C}$ for $6 \mathrm{~h}$ under various atmospheres $\left(\mathrm{Ar}, \mathrm{N}_{2}\right.$, or $\mathrm{Ar}$ with $5 \%$ $\mathrm{H}_{2}\left(\mathrm{ArW}_{5}\right)$ ) was undertaken with a ramp of $13^{\circ} \mathrm{C} \cdot \mathrm{min}^{-1}$ and a flow rate of $20 \mathrm{~mL} \cdot \mathrm{min}^{-1}$. The oven was then cooled down slowly $(10 \mathrm{~h})$ to room temperature. In total, five samples were prepared: almond precursor synthesized under an Ar atmosphere (ALM_AR), almond precursor synthesized under a $\mathrm{N}_{2}$ atmosphere (ALM_N2), almond precursor synthesized under an $\mathrm{Ar} / \mathrm{H}_{2}$ atmosphere (ALM_ARW5), walnut precursor synthesized under an Ar atmosphere (WN_AR), and fir precursor synthesized under an Ar atmosphere (FIR_AR).

Electrochemical measurements.-Slurries were prepared from a mixture of $90 \mathrm{wt} \%$ carbonaceous material and $10 \mathrm{wt} \%$ 

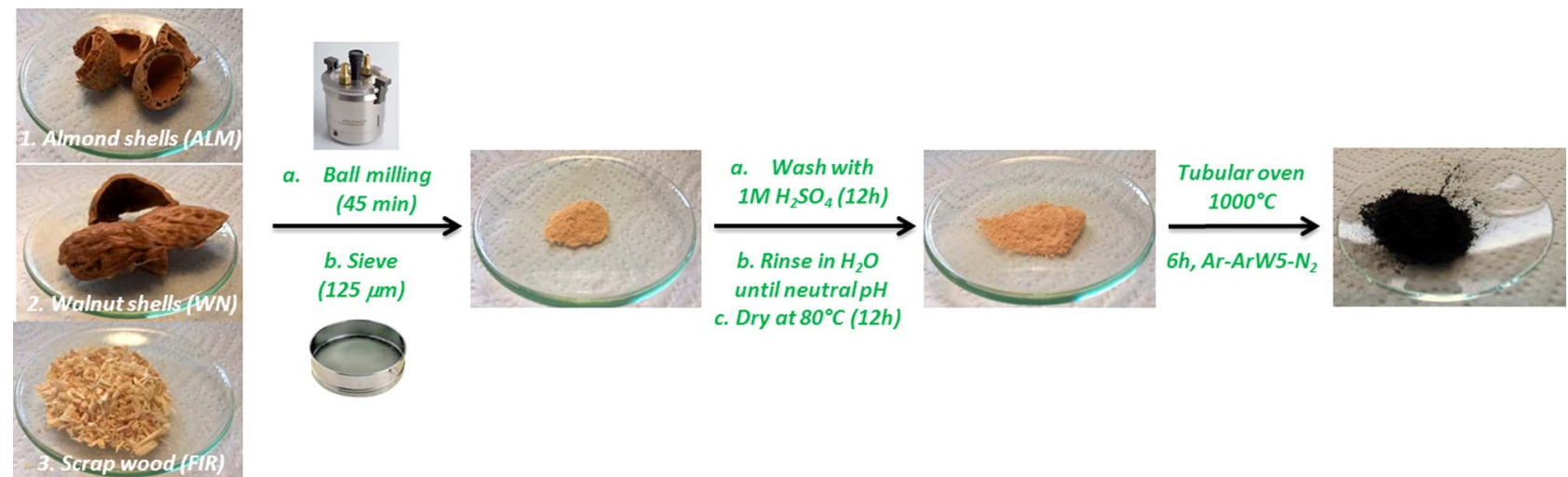

Figure 1. Synthesis protocol used to obtain carbonaceous materials.

carboxymethylcellulose sodium salt (CMC; Alfa Aesar) dispersed in a solution of deionized water and ethanol $(4: 1, \mathrm{v} / \mathrm{v})$. The obtained ink was cast onto an aluminum foil (used as the current collector), dried under air at room temperature, and calendared at a thickness of $80 \mu \mathrm{m}$. Electrodes (diameter: $13 \mathrm{~mm}$ ) with an active material loading of around $5 \mathrm{mg} \cdot \mathrm{cm}^{-2}$ were punched and dried under dynamic vacuum at $80^{\circ} \mathrm{C}$ for $12 \mathrm{~h}$. Electrochemical cells were assembled in an Ar-filled glove box using a glass fiber separator and metallic sodium as the counter electrode. The electrolyte consisted of $1 \mathrm{M} \mathrm{NaPF}_{6}$ dissolved in a mixture of ethylene carbonate (EC) and diethyl carbonate (DEC) (1:1) (Kishida Chemical). Using an Astrol cycling device in galvanostatic mode, the cells were cycled between $5 \mathrm{mV}$ and $1.1 \mathrm{~V}$ (versus $\left.\mathrm{Na}^{+} / \mathrm{Na}\right)$ at a $\mathrm{C} / 10$ rate $(10 \mathrm{~h}$ for a full charge or discharge considering a theoretical specific charge of $\left.300 \mathrm{mAh} \cdot \mathrm{g}^{-1}\right)$ at $25^{\circ} \mathrm{C} .{ }^{11}$ Potentiostatic steps of $1 \mathrm{~h}$ and $30 \mathrm{~min}$ were added at the end of each discharge and charge respectively. In the text, all the potentials are expressed relative to $\mathrm{Na}$ metal (vs. $\mathrm{Na}^{+} / \mathrm{Na}$ ).

XRD measurements.-XRD measurements were performed in capillary mode at $25^{\circ} \mathrm{C}$ with a PANalytical Empyrean diffractometer using $\mathrm{Cu} \mathrm{K} \alpha$ radiation and $0.3 \mathrm{~mm}$ diameter glass capillaries. The patterns are the sum of 13 diffractograms, each acquired over $1 \mathrm{~h}$ from $10^{\circ}$ to $60^{\circ}$ with a step size of $0.0334^{\circ}$.

SEM measurements.-SEM measurements were performed using a Carl Zeiss Ultra55 scanning electron microscope in both the secondary electron mode and the InLens mode at $3 \mathrm{kV}$.

BET specific surface area measurements.-The porosities of the carbonaceous materials were determined from their $\mathrm{N}_{2}$ adsorption/desorption isotherms at $77 \mathrm{~K}$ (liquid nitrogen) with a BETAutosorb-1C AX1C-MP-LP instrument (Quantachrome Instruments) using the BET theory. The samples were degassed under vacuum for $12 \mathrm{~h}$ at $250^{\circ} \mathrm{C}$. The BET surface specific area was estimated in the relative pressure range $\left(\mathrm{P} / \mathrm{P}_{0}\right)$ of $10^{-2}$ to $10^{-1}$ with a $\mathrm{P}_{0}$ value of $741.23 \mathrm{mmHg}$. The particle size distribution was obtained from the adsorption branch of the isotherm using density functional theory (DFT) methods.

Raman spectroscopy.-The Raman spectra were acquired using a Labram HR800 Raman microscope (Horiba-Jobin Yvon) equipped with a He-Ne laser $(632.8 \mathrm{~nm})$. Each spectrum is the sum of four spectra, each acquired over $25 \mathrm{~s}$ from $800 \mathrm{~cm}^{-1}$ to $1900 \mathrm{~cm}^{-1}$. After subtracting the background, the data were fitted with five Lorentzian peaks (D1, D2, D3, D4, and G) using the CasaXPS software (Casa Software Ltd).

SAXS measurements.-Samples for SAXS measurements were prepared by placing powder between two pieces of tape to obtain a very thin pellet-like specimen. The measurements were performed at
$9 \mathrm{~kW}(45 \mathrm{mV}$ times $200 \mathrm{~mA})$ in transmission mode, between a $\mathrm{q}$ value of $0.05 \mathrm{~nm}^{-1}$ and $10 \mathrm{~nm}^{-1}$, using a Rigaku Smartlab diffractometer equipped with a copper rotating anode and a Hy-Pix 3000 array detector. A graphite Göbel mirror was used to remove the $\mathrm{Cu}$ $\mathrm{K}_{\beta}$ radiation and to make the $\mathrm{X}$-ray beam parallel. A collimator with a $20 \mu \mathrm{m}$ hole was placed along the X-ray path to reduce the area of the beam spot on the sample. The SAXS measurements were conducted by exposing the sample to X-rays for $900 \mathrm{~s}$. The contribution from the tape was removed. The 2DP software from Rigaku was used for radial integration of the data and subtraction of the calculated contribution from $\mathrm{Cu} \mathrm{K \alpha} \alpha_{2}$. Linear fits of the SAXS curves were performed in the q-range of $0.1-0.3 \mathrm{~nm}^{-1}$ using OriginLab, whereas fits in the q-range of $1-6 \mathrm{~nm}^{-1}$ were performed using SASFit software. ${ }^{12}$

\section{Results and Discussion}

Effect of gas annealing $\left(\mathrm{Ar}, \mathrm{N}_{2}\right.$, and $\left.\mathrm{Ar} \mathrm{W}_{5}\right)$.-Characterization of the carbonaceous materials.-Using the synthesis protocol displayed in Figure 1, ALM samples were calcined under various atmospheres. Following calcination, the obtained carbonaceous materials were investigated by SEM (Figure 2), which revealed that the samples had similar morphological features, independent of the atmosphere used. Typically, the carbonaceous materials consisted of a mixture of primary particles with sizes ranging from $4 \mu \mathrm{m}$ to $10 \mu \mathrm{m}$ and agglomerates of up to $50 \mu \mathrm{m}$. Surprisingly, the initial morphology was maintained upon calcination. At higher magnification, some spherical structures of less than $1 \mu \mathrm{m}$ in diameter were observed on the surfaces of the primary particles. These spherical structures were either closed or opened. Similar features have been observed on pyrolyzed leaves ${ }^{8}$ and walnut shells. ${ }^{13}$ According to the literature, these macropores improve the wettability of the material. The presence of these localized macropores might be explained by the fast heating ramp coupled with the gases released during calcination $\left(\mathrm{CO}, \mathrm{CO}_{2}\right)$. Additionally, the sample calcined under $\mathrm{Ar} / \mathrm{H}_{2}$ (ALM_ARW5, Figure 2c) exhibited some areas of open porosity (pores of tens of nanometers) on the surface of the particles (indicated by white circles in Figure 2c).

The XRD patterns of the synthesized carbonaceous samples (Figure 3a) are characteristic of amorphous carbons with graphitic domains ( $\mathrm{sp}^{2}$ domains). ${ }^{14,15}$ The two broad Bragg peaks at $22.0^{\circ}$ and $43.7^{\circ}$ were indexed to the (002) and (100) planes, respectively, which both belong to the graphite hexagonal structure. The XRD patterns of the ALM_AR and ALM_N2 samples are identical. By using the Bragg's law $(2 \mathrm{~d} \sin (\theta)=\lambda)$, a similar interlayer distance $\left(\mathrm{d}_{002}=4.0 \AA\right)$ was found for all the samples, independently of the annealing gas used. This value is in agreement with those reported in the literature for carbons obtained from biomass. ${ }^{5,10}$ Moreover, the stacking height Lc and the lateral size La of the crystallites, calculated using the Scherrer equation, ${ }^{16}$ are close for all the carbonaceous materials (Table I). 

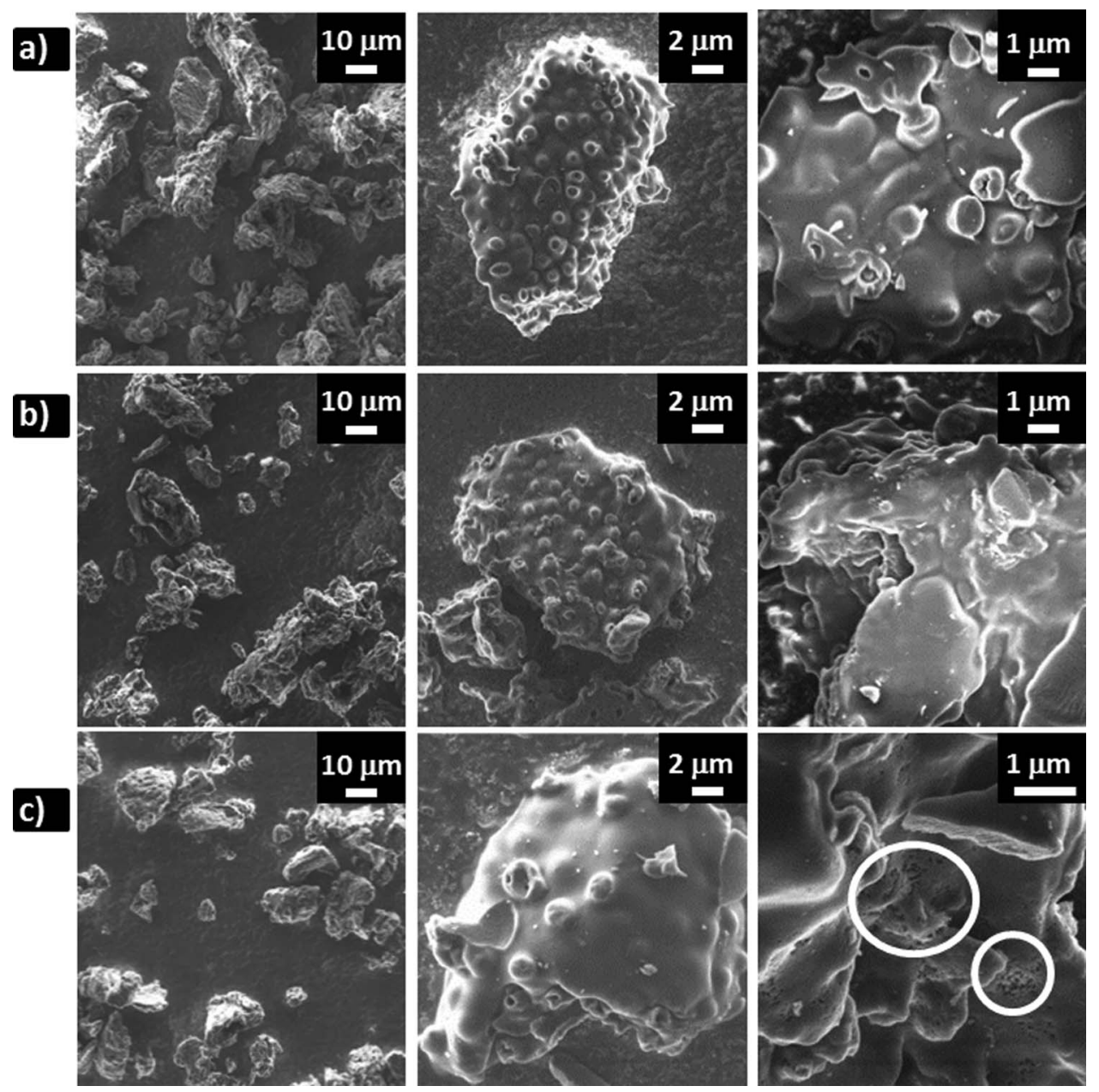

Figure 2. SEM images at different magnifications of (a) ALM-AR, (b) ALM_N2, and (c) ALM_ARW5 materials.

The normalized Raman spectra of the carbonaceous materials are displayed in Figure $3 \mathrm{~b}$. Each spectrum is characterized by two main broad bands, the $\mathrm{D}$ and $\mathrm{G}$ bands, located at $1332 \mathrm{~cm}^{-1}$ and $1597 \mathrm{~cm}^{-1}$, respectively. The $\mathrm{D}$ band, which is the most intense peak, revealed high disorder in the carbon structure, whereas the $\mathrm{G}$ band indicated the presence of graphitic domains in the carbonaceous samples. ${ }^{17-19}$ The annealing gas had no effect on the Raman spectral signature. However, the spectrum of ALM_ARW5 showed slightly narrower D and $G$ bands than those of ALM_AR and ALM_N2 (Figure 3b).

According to Ferrari et al., ${ }^{1 \overline{8}}$ there is no common methodology to deconvolute the Raman spectra of disordered carbon materials. Sadezky et al. ${ }^{20}$ tested several deconvolution pathways with three or five contributions, and achieved the most accurate results using five Lorentzian peaks (G, D1, D2, D3, and D4). The D1 band centered around $1350 \mathrm{~cm}^{-1}$ was indexed to the vibration mode of microcrystalline graphite $\left(\mathrm{A}_{1 \mathrm{~g}}\right.$ symmetry) and the $\mathrm{D} 2 \mathrm{band}$, found at $1620 \mathrm{~cm}^{-1}$, was indexed to a vibration mode of a disordered graphitic lattice $\left(\mathrm{E}_{2 \mathrm{~g}}\right.$ symmetry). Moreover, in addition to the $\mathrm{G}$ band (ideal graphitic lattice, $E_{2 g}$ symmetry), the D3 band around $1500 \mathrm{~cm}^{-1}$ and D4 band around $1200 \mathrm{~cm}^{-1}$ were attributed to an amorphous carbon contribution (including adsorbed molecules or molecular fragments) ${ }^{21}$ and to an $\mathrm{sp}^{3}$-rich phase, ${ }^{19}$ respectively. We applied the aforementioned deconvolution methodology to our systems, as shown in Figure $3 \mathrm{c}$ for ALM_AR. The peak area ratios of $A_{D 1} / A_{G}, A_{D 3} / A_{G}$, and $A_{D 4} / A_{G}$ are reported in Table I. The $\mathrm{A}_{\mathrm{D} 1} / \mathrm{A}_{\mathrm{G}}$ and $\mathrm{A}_{\mathrm{D} 4} / \mathrm{A}_{\mathrm{G}}$ ratios of all the carbonaceous materials were similar (around 3.7 and 0.7 , respectively), independent of the annealing gas employed. However, the $A_{D 3} / A_{G}$ ratio decreased slightly in the case of ALM_ARW5 (0.8) relative to that for the other materials (1.1). Therefore, it can be assumed that adsorbed molecules on the surface of ALM_ARW5 are less important, most probably because they were reduced during the annealing process.

To investigate the porosity of the carbonaceous materials, $\mathrm{N}_{2}$ adsorption/desorption isotherms were obtained, as shown in Figures $4 \mathrm{a}-4 \mathrm{c}$. The adsorption isotherms were all of type IV and the observed hystereses corresponded to type $\mathrm{H} 4,{ }^{22}$ which implied that the materials have micro-/mesoporous properties. ${ }^{23}$ The pore size distributions obtained from BET measurements (Figure 4d) further confirmed the presence of micropores $(<2 \mathrm{~nm})$ and mesopores $(2-5 \mathrm{~nm})$ in the ALM_N2 and ALM_ARW5 samples. The specific surface areas of these samples were estimated as $185 \mathrm{~m}^{2} \cdot \mathrm{g}^{-1}$ and $252 \mathrm{~m}^{2} \cdot \mathrm{g}^{-1}$ respectively. The results obtained for the ALM_ARW5 sample are supported by the SEM analysis. Unexpectedly, no micropores were detected in the ALM_AR sample (absence of a drop at very low $\mathrm{P} / \mathrm{P}_{0}$ in the isotherm (Figures 4a)), resulting in a low specific surface area of $21 \mathrm{~m}^{2} \cdot \mathrm{g}^{-1}$. This result is not unexpected, as it has been reported that microporosity and ultramicroporosity $(<0.7 \mathrm{~nm})$ may not be fully accessible to the $\mathrm{N}_{2}$ gas used for BET measurements. ${ }^{24-26}$

Thus, we conducted SAXS experiments to further examine the porosity of the carbonaceous samples ${ }^{27}$ (Figures $4 \mathrm{e}-4 \mathrm{f}$ ). The obtained scattering curves (Figure 4e) are typical of carbon samples. ${ }^{28}$ The linear data between 0.1 and $0.7 \mathrm{~nm}^{-1}$ were fitted to the power law $\mathrm{I} \propto \mathrm{q}^{-\alpha}$ to calculate the surface fractal, Ds $=6-\alpha$. The scattering curve profile in the range of $0.7-10 \mathrm{~nm}^{-1}$ is related to the ultramicroporosity of the material. These segments were fitted by assuming spherical pores with radii that follow a log-normal size distribution. Based on these results, the ALM_AR and ALM_N2 samples have similar porosities. The surface fractal was found to be 2.3 and 2.4 for 

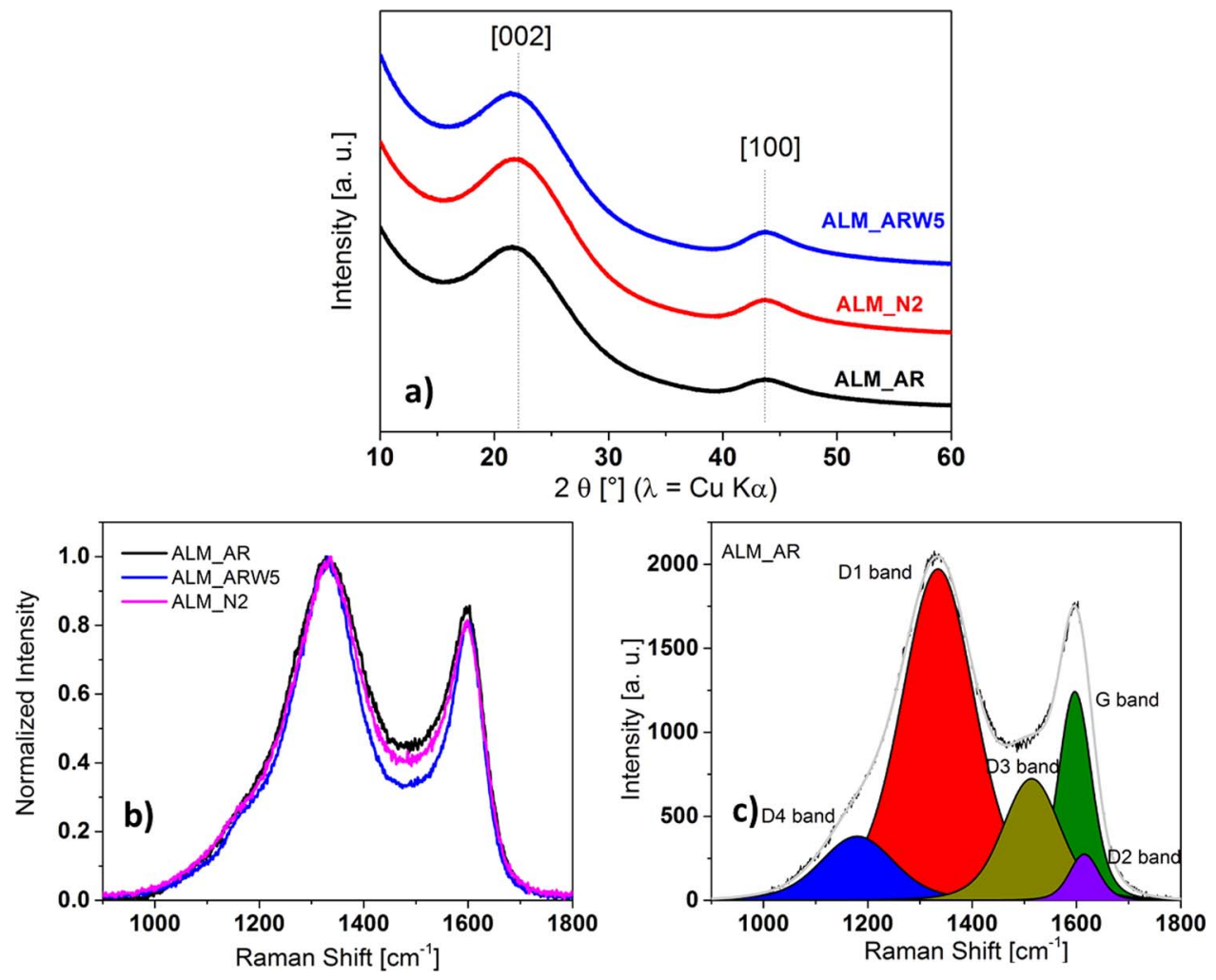

Figure 3. a) XRD patterns of the carbonaceous materials synthesized under various atmospheres; b) Raman spectra of the carbonaceous materials and c) proposed deconvolution of the Raman spectrum of ALM_AR.

ALM_AR and ALM_N2, respectively, in agreement with the values reported in the literature for carbon black with a random arrangement of crystallites. ${ }^{29,30}$ Surprisingly, the surface fractal increases considerably to 2.9 for the ALM_ARW5 sample, which is comparable to the values for activated carbons. ${ }^{31}$ The pore size distributions (Figure 4f) confirmed the presence of ultramicropores in all the samples and, therefore, it can be assumed that the micropores of ALM_AR were not accessible to $\mathrm{N}_{2}$ gas molecules (the pores of this particular sample may not be spherical), resulting in an incorrect value for the BET surface area. Additionally, the significant difference in the intensities of the main peak centered around $0.3 \mathrm{~nm}$ in the SAXS pore size distribution curves indicated that the number of pores in the samples decreased in the following order: ALM_ARW5 (including closed porosity) > ALM_AR > ALM_N2.

Electrochemical performance of almond-based materials.-The electrochemical performance of the carbonaceous materials measured at a rate of $\mathrm{C} / 10$ is presented in Figure 5. In the first cycle, similar specific charge of $313 \mathrm{mAh} \cdot \mathrm{g}^{-1}$ and $318 \mathrm{mAh} \cdot \mathrm{g}^{-1}$ were obtained for ALM_AR and ALM_N2, respectively, whereas ALM_ARW5 only achieved a specific charge of $270 \mathrm{mAh} \cdot \mathrm{g}^{-1}$. As expected owing to solid electrolyte interphase (SEI) formation, the specific charges decreased in the $2^{\text {nd }}$ cycle to $260 \mathrm{mAh} \cdot \mathrm{g}^{-1}$ (ALM_N2) and $252 \mathrm{mAh} \cdot \mathrm{g}^{-1}$ (ALM_AR), and were then stable for the next 60 cycles. These specific charges are close to the literature values for electrode made from biomass. ${ }^{4,9}$ Interestingly, the sample synthesized under a reductive atmosphere (ALM_ARW5) provided a lower reversible specific charge of only ca. $128 \mathrm{mAh} \cdot \mathrm{g}^{-1}$. Although the Coulombic efficiency of each sample during the $1^{\text {st }}$ cycle was ca. $80 \%$, the Coulombic efficiencies of the subsequent cycles were higher than $98.5 \%$ for ALM_AR and ALM_N2 and around 96\% for ALM_ARW5.

After 60 cycles, the electrochemical performance of ALM_AR and ALM_N2 deteriorated considerably, with specific charges close to $200 \mathrm{mAh} \cdot \mathrm{g}^{-1}$. However, stable Coulombic efficiencies were maintained. This phenomenon is attributed to resistance buildup in the system. A slight decrease in the specific charge was also observed for the ALM_ARW5 sample after 70 cycles.

The galvanostatic curves for the $1^{\text {st }}$ and $2^{\text {nd }}$ cycles of the carbonaceous samples are shown in Figure 6. The ALM_AR and ALM_N2 samples displayed similar profiles for both cycles (Figure 6). The observed profile is typical of hard carbon materials. ${ }^{32}$ During the $1^{\text {st }}$ discharge, a small shoulder (labeled I in Figure 6a) appeared during the early sodiation stage between $1.0 \mathrm{~V}$ and $0.8 \mathrm{~V}$, accounting for $20 \%$ of the total specific charge. This feature is related to electrolyte decomposition, which is an irreversible process. Then, the potential profile can be divided in two parts: a sloping region between $0.8 \mathrm{~V}$ and $80 \mathrm{mV}$ (labeled II in the Figure 6a) and a potential plateau at $80 \mathrm{mV}$ (labeled III in Figure 6a). The former region was assigned to

\section{Table I. Properties of the carbonaceous materials. The asterisk indicates that this value is not true according to the results of the SAXS experiment} (see SAXS part).

\begin{tabular}{cccccccr} 
Sample & $\mathrm{Lc}[\mathrm{nm}]$ & $\mathrm{La}[\mathrm{nm}]$ & $\mathrm{A}_{\mathrm{D} 1} / \mathrm{A}_{\mathrm{G}}$ ratio & $\mathrm{A}_{\mathrm{D} 3} / \mathrm{A}_{\mathrm{G}}$ ratio & $\mathrm{A}_{\mathrm{D} 4} / \mathrm{A}_{\mathrm{G}}$ ratio & Surface area $\left[\mathrm{m}^{2} \cdot \mathrm{g}^{-1}\right]$ & Surface fractal, Ds \\
\hline ALM_AR & 0.8 & 2.9 & 3.7 & 1.1 & 0.7 & $21^{*}$ & 2.3 \\
ALM_N2 & 0.8 & 2.9 & 3.7 & 1.0 & 0.7 & 2.7 & 2.7 \\
ALM_ARW5 & 0.8 & 3.0 & 3.6 & 0.8 & 0.7 & 8.9 & not measured \\
WN_AR & 0.8 & 3.0 & 3.7 & 1.1 & 0.7 & 30 & not measured
\end{tabular}



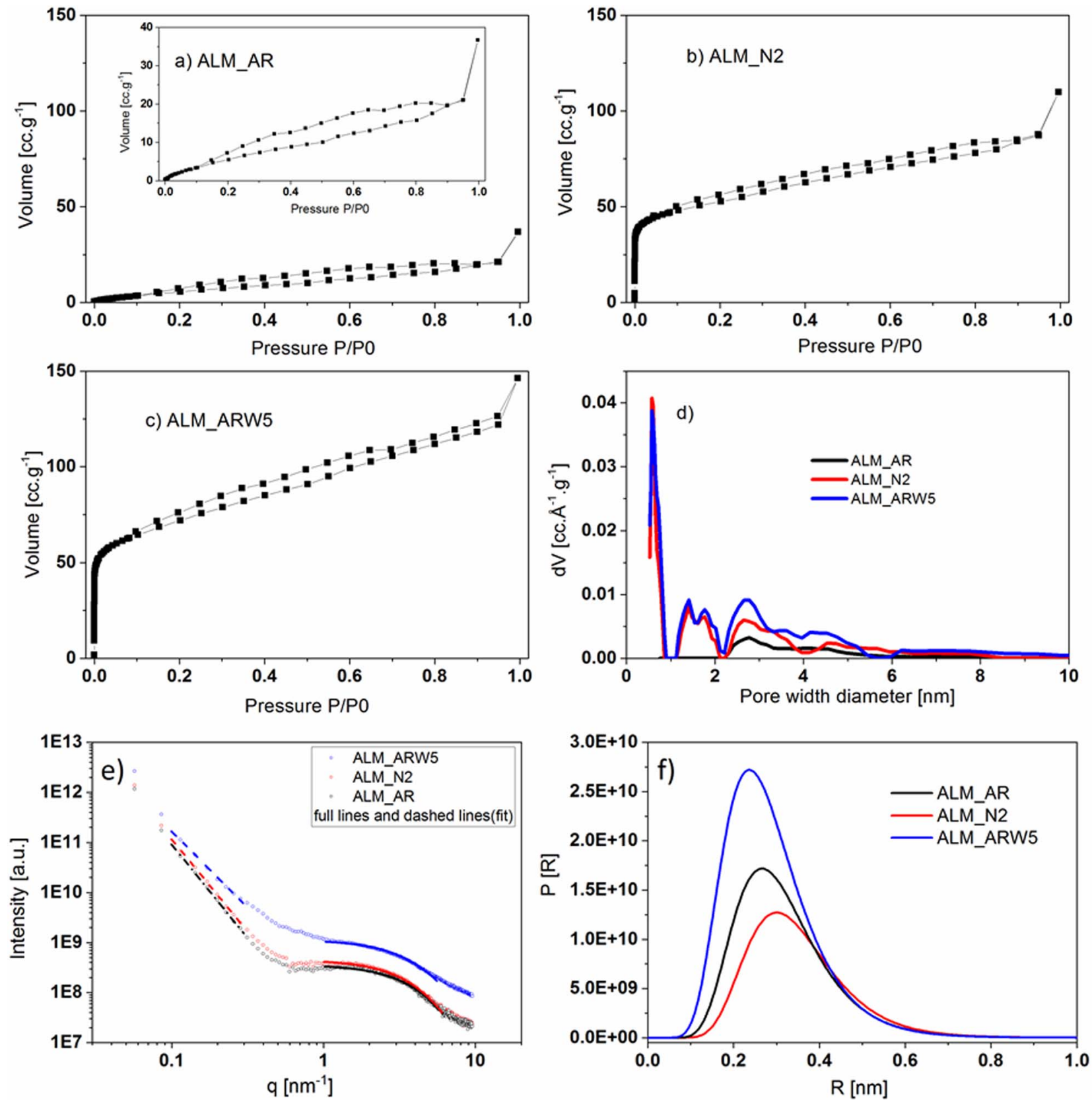

Figure 4. $\mathrm{N}_{2}$ adsorption/desorption isotherms of a) ALM_AR, b) ALM_N2, and c) ALM_ARW5, and d) corresponding BET pore size distributions of the carbonaceous materials. e) SAXS data for the carbonaceous materials and $\mathrm{f}$ ) the corresponding pore size distribution curves following log-normal distributions.

$\mathrm{Na}$ adsorption in defect sites or disordered graphene layers and the latter region was attributed to filling of the nanopores by $\mathrm{Na}$ ions. ${ }^{32}$ The length of the potential plateau (III) may be correlated to the total porous volume ${ }^{33}$ and especially to the mesopores. ${ }^{34}$ However, a recent study by Ghimbeu et al. ${ }^{26}$ indicated that this assumption might be not correct owing to difficulties in measuring the microporosity by $\mathrm{N}_{2}$ adsorption and BET analysis. Instead, they proposed that the sloping curve could be attributed to filling of the nanopores, whereas the potential plateau could correspond to insertion of $\mathrm{Na}$ ions into the graphitic planes.

During the $1^{\text {st }}$ discharge of the ALM_ARW5 sample, the shoulder related to electrolyte decomposition was considerably less pronounced and could only be detected in the derivative plot (Figure 6b), with a broad peak from $0.35 \mathrm{~V}$ to $0.15 \mathrm{~V}$. This phenomenon might be related to differences in the surface groups of this sample (cf. D3 band, Raman experiment), which would strongly influence the passivation layer. ${ }^{15,32}$ However, regions II and III of the ALM_ARW5 sample are similar to those for the ALM_AR and ALM_N2 samples, but the length of the potential plateau (region III) is shorter. This feature can be observed in the derivative of the galvanostatic curve (Figure 6b), in which the area of peak related to region III is less pronounced for ALM_ARW5. Interestingly, despite the potential plateau starting at a higher potential (ca. $0.2 \mathrm{~V}$ ), a smaller polarization is observed in the galvanostatic curve (Figure 6a) compared with that of the other carbonaceous samples. This difference might be due to the high porosity of ALM_ARW5, which promoted higher electrolyte decomposition, resulting in additional resistance in the system.

The desodiation of ALM_AR and ALM_N2 proceeded through a potential plateau at $100 \mathrm{mV}$ (labeled I' in Figure 6a) followed by a sloping region until $1.1 \mathrm{~V}$ (labeled II' in Figure 6a). For the ALM_ARW5 sample, the potential plateau at $100 \mathrm{mV}$ overlapped with those observed for the ALM_AR and ALM_N2 samples, but contributed to 


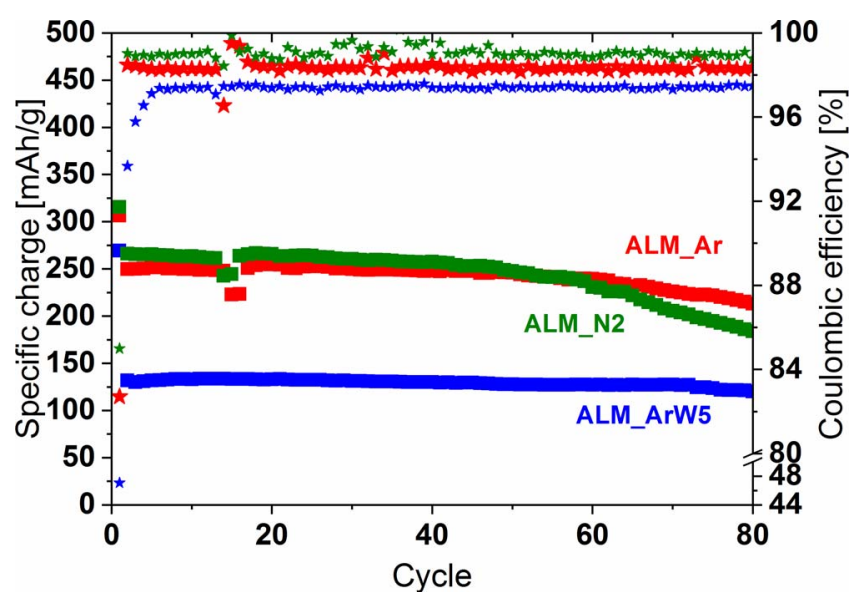

Figure 5. Electrochemical performance of the carbonaceous materials cycled at a rate of $\mathrm{C} / 10$ in an electrolyte of $1 \mathrm{M} \mathrm{NaPF}_{6}$ in EC/DEC.

only $45 \%$ of the total specific c compared with $60 \%$ for ALM_AR and ALM_N2. We believe that the higher extent of electrolyte decomposition during the discharge process probably hindered the access to some pores, resulting in decreased insertion of Na in ALM_ARW5 relative to ALM_AR and ALM_N2.

During the $2^{\text {nd }}$ cycle (Figure $6 \mathrm{c}$ ), the shoulder assigned to electrolyte decomposition (region I) was no longer observed, but the other two regions (II and III for discharge and $\mathrm{I}^{\prime}$ and $\mathrm{II}^{\prime}$ for charge) were still present. As the physical and morphological properties of the ALM_AR and ALM_N2 samples were similar, the near overlap of the observed electrochemical signatures could be anticipated. Moreover, a small peak at ca. $100 \mathrm{mV}$ before the main peak at ca. $70 \mathrm{mV}$ was detected in the derivative curves (Figure 6b). Wu et al. ${ }^{6}$ assigned this feature to enhanced electrode wetting and breathing of the electrode corresponding to sodiation/desodiation processes upon cycling.

Thus, we established a low-cost synthesis protocol to obtain carbonaceous materials. We demonstrated that the annealing gas has consequences on the electrochemical performance, especially when a reductive atmosphere is used; however, the use of $\mathrm{N}_{2}$ gas could be a very good compromise and cheap solution. We subsequently investigated the impact of the type of lignin-based precursor on the properties and electrochemical performance of the carbonaceous material where Ar gas was arbitrary used.

Effect of different lignin-based precursors.-Characterization of the carbonaceous materials. - In addition to ALM, WN and FIR were used as lignin-based precursors under an Ar atmosphere (WN_AR and FIR_AR, respectively). The morphology and the particle size distribution of the WN_AR sample were identical to those of ALM_AR (Figures $7 \mathrm{a}$ and $7 \mathrm{~b}$ ), with primary particle sizes in the range of $4-10 \mu \mathrm{m}$ and some agglomerates of up to $50 \mu \mathrm{m}$. However, instead of the spherical features observed on the surface of ALM_AR, open pores of $0.5-1 \mu \mathrm{m}$ in size were observed on the surface of WN_AR. Moreover, the surface of the WN_AR particles appeared to be covered in additional small fiber-like features. In contrast, the FIR_AR sample displayed a fiber-like morphology (Figure 7c) with a similar particles size compared to ALM_AR and WN_AR. Unlike the big round aggregates of ALM_AR and WN_AR, the fiber-like or needle-like morphology might have a beneficial impact of the insertion/de-insertion of $\mathrm{Na}$ by reducing the transport length. At higher magnification, the
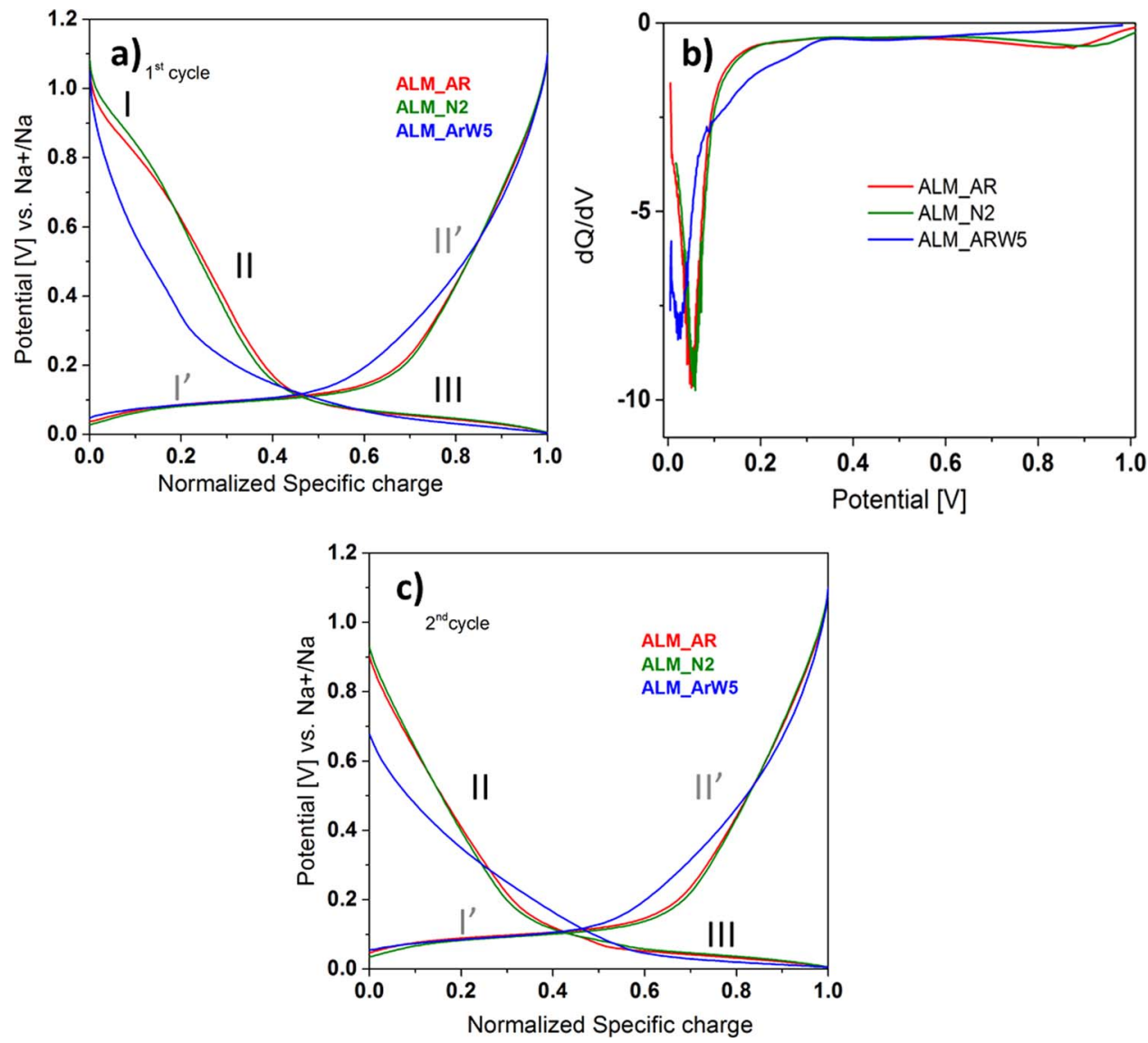

Figure 6. Galvanostatic curves for (a) the $1^{\text {st }}$ cycle, with b) its derivative curves, and (c) the $2^{\text {nd }}$ cycle of the various carbonaceous materials cycled at a rate of $\mathrm{C} / 10$ in an electrolyte of $1 \mathrm{M} \mathrm{NaPF}_{6}$ in EC/DEC 

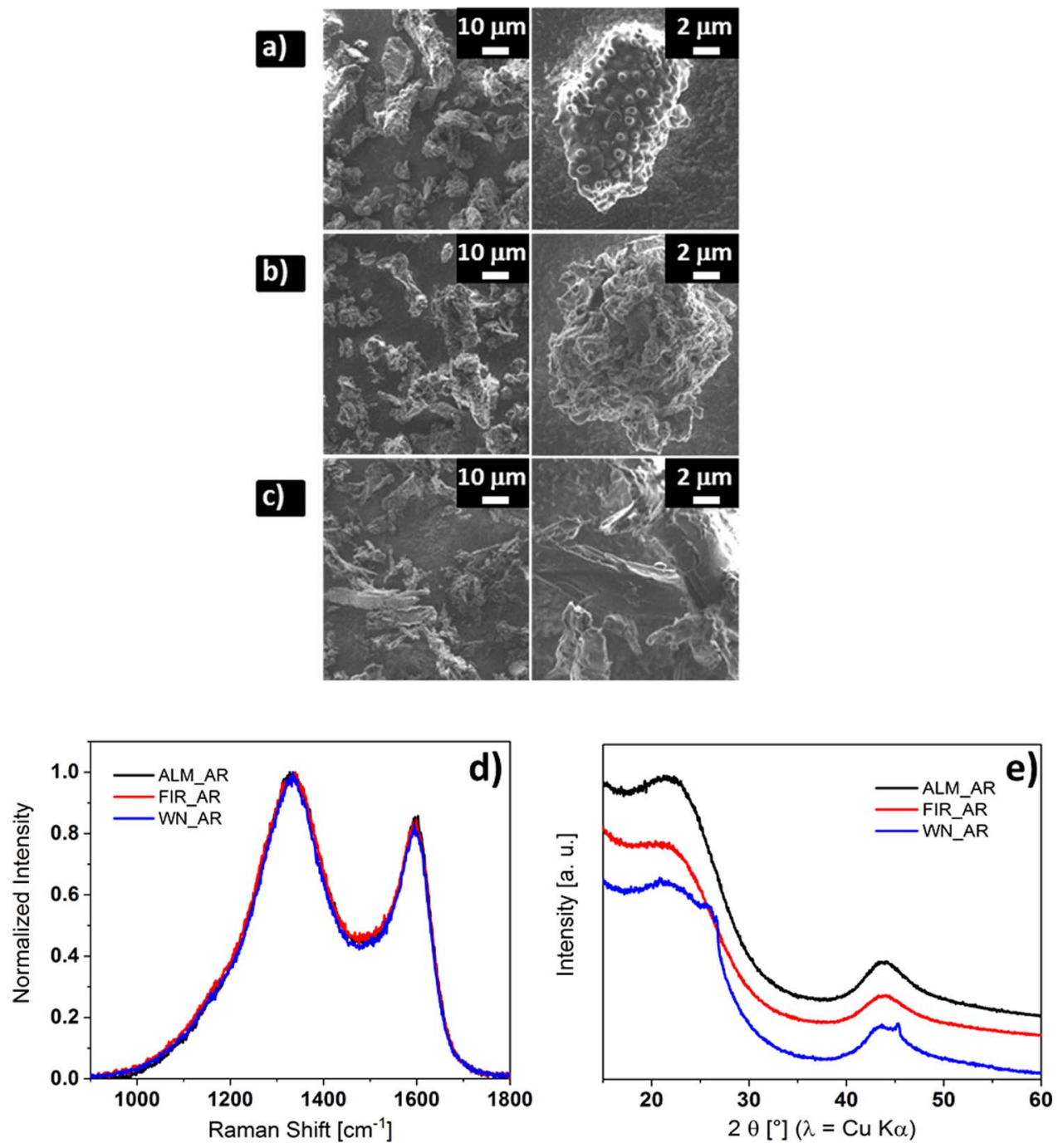

Figure 7. SEM images of (a) ALM_AR, (b) WN_AR, and (c) FIR_AR samples; d) Raman spectra and e) XRD patterns of the carbonaceous materials synthesized from different lignin-based precursors.

surface of the carbonaceous FIR_AR material appeared clean without any specific features.

The results obtained from Raman and BET measurements are summarized in Table I. Similar $\mathrm{d}_{002}$ values and $\mathrm{A}_{\mathrm{Dx}} / \mathrm{A}_{\mathrm{G}}(\mathrm{x}=1$ and 4$)$ ratios were obtained for all the carbonaceous materials, independent of the type of precursor used. This similarity is confirmed by the identical Raman spectra obtained for the three lignin-based precursors (Figure 7d). The XRD pattern of WN_AR (Figure 7f) revealed the presence of a small amount of an impurity compared with the other samples. Specific surface areas of $88 \mathrm{~m}^{2} \cdot \mathrm{g}^{-1}$ and $30 \mathrm{~m}^{2} \cdot \mathrm{g}^{-1}$ were determined for WN_AR and FIR_AR, respectively, confirming the trend observed for ALM_AR.

Electrochemical performance of materials from other lignin-based precursors.-The electrochemical performance of the materials prepared from different lignin-based precursors are summarized in Figure 8a. Similar specific charges $\left(308-320 \mathrm{mAh} \cdot \mathrm{g}^{-1}\right)$ were reached during the $1^{\text {st }}$ cycle. The specific charges decreased to $270 \mathrm{mAh} \cdot \mathrm{g}^{-1}$ for FIR_AR and $252 \mathrm{mAh} \cdot \mathrm{g}^{-1}$ for both ALM_AR and WN_AR upon further cycling. As previously mentioned, the drop in specific charge is due to electrolyte decomposition, which seemed identical for all lignin-based precursors. During the 60 first cycles, the specific charges of the WN_AR and ALM_AR samples remained similar at ca. $250 \mathrm{mAh} \cdot \mathrm{g}^{-1}$ with a Coulombic efficiency of around $98.5 \%$.
Interestingly, the FIR AR sample sustained a specific charge of ca. $270 \mathrm{mAh} \cdot \mathrm{g}^{-1}$ for 80 cycles with a slightly higher Coulombic efficiency of ca. $99.5 \%$. The galvanostatic curves of all three materials were similar (Figures 8b). In addition, we can see that the current stabilizes faster with FIR_AR sample at the end of the $2^{\text {nd }}$ and $20^{\text {th }}$ sodiation than for the ALM sample indicating that the Na pathway is favorited by the morphology in FIR sample (Figures 8c-8d).

These results indicate that the morphologies of the precursors, which is the only different parameter found for FIR_AR in comparison with ALM_AR and WN_AR, have a small but important effect on the electrochemical performance. Despite a similar overall performance (specific charges of 250-270 $\mathrm{mAh} \cdot \mathrm{g}^{-1}$ ), the fiber-like morphology of FIR may promote access to the graphene sheets compared with the other materials, leading to greater stability and a higher specific charge. Bai et al. ${ }^{35,36}$ observed similar results when comparing fiber like morphology and round shape particles of carbon materials.

\section{Conclusions}

Carbonaceous materials were synthesized from ALM under various inert atmospheres ( $\mathrm{Ar}, \mathrm{N}_{2}$, and $\left.\mathrm{Ar} / \mathrm{H}_{2}\right)$. Characterization by XRD, SEM, and Raman spectroscopy revealed similar physical and morphological properties for the materials synthesized under $\mathrm{Ar}$ and $\mathrm{N}_{2}$ atmospheres, resulting in similar electrochemical performance. Typically, 

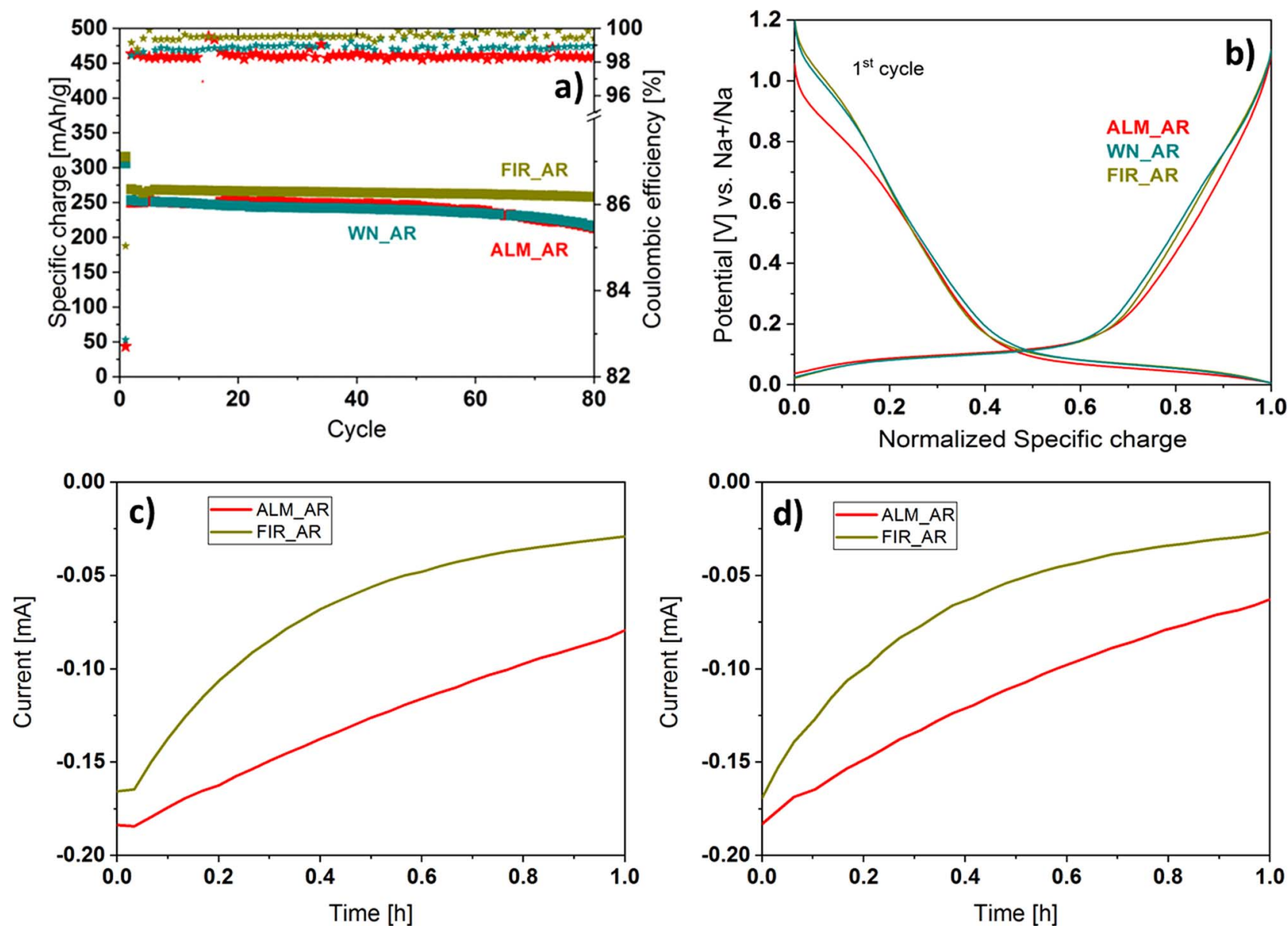

Figure 8. a) Electrochemical performance and b) Galvanostatic curves for the $1^{\text {st }}$ cycle of the carbonaceous materials synthesized from different lignin-based precursors cycled at a rate of $\mathrm{C} / 10$ in an electrolyte of $1 \mathrm{M} \mathrm{NaPF}_{6}$ in EC/DEC; Evolution of the current during the potentiostatic step at $5 \mathrm{mV}$ for the c) $2^{\text {nd }}$ and d) $20^{\text {th }}$ sodiation.

a specific charge of ca. $255 \mathrm{mAh} \cdot \mathrm{g}^{-1}$ was achieved with long-term stability. In contrast, the carbonaceous material synthesized under reductive conditions $\left(\mathrm{Ar} / \mathrm{H}_{2}\right)$ exhibited poor electrochemical performance owing to a higher porosity and different surface chemistry. Furthermore, we demonstrated that the use of different precursors led to similar electrochemical performance. However, a fibrous-like morphology helped to i) stabilize the specific charge during long-term cycling and ii) improve the electrochemical performance (specific charge of $270 \mathrm{mAh} \cdot \mathrm{g}^{-1}$ ). Thus, we demonstrated that low-cost electrode materials with reasonable electrochemical performance can be developed using a cheap and reliable synthesis procedure.

\section{Acknowledgment}

This work was performed within the SCCER Heat and Storage Network (Swiss Competence Center for Heat and Electricity Storage). The authors thank Eibar Flores for the help with the Raman measurements, Dr Sigita Trabesinger and Dr. Joanna Conder for the helpful discussion about BET measurements.

\section{ORCID}

Cyril Marino (D) https://orcid.org/0000-0002-6258-5636

\section{References}

1. D. Hoornweg, P. Bhada-Tata, and C. Kennedy, Nature., 502, 615 (2013).

2. E. Schröder, K. Thomauske, B. Oechsler, and S. Herberger, in Activated Carbon from Waste Biomass, Vol. (Ed. D. S. Shaukat,), 333 (2011).
3. J. Górka, C. Vix-Guterl, and C. Matei Ghimbeu, Journal of Carbon Research., 2, 24 (2016).

4. X. Dou, I. Hasa, M. Hekmatfar, T. Diemant, R. Jürgen Behm, D. Buchholz, and S. Passerini, ChemSusChem., 10, 2668 (2017).

5. E. M. Lotfabad, J. Ding, K. Cui, A. Kohandehghan, W. P. Kalisvaart, M. Hazelton, and D. Mitlin, ACS Nano., 8, 7115 (2014).

6. L. Wu, D. Buchholz, C. Vaalma, G. A. Giffin, and S. Passerini, ChemElectroChem. 3, 292 (2016).

7. K.-I. Hong, L. Qie, R. Zeng, Z.-Q. Yi, W. Zhang, D. Wang, W. Yin, C. Wu, Q.-J. Fan, W.-X.Zhang, and Y.-H. Huang, Journal of Materials Chemistry A., 2, 12733 (2014).

8. H. Li, F. Shen, W. Luo, J. Dai, X. Han, Y. Chen, Y. Yao, H. Zhu, K. Fu, E. Hitz, and L. Hu, ACS Appl Mater Interfaces., 8, 2204 (2016).

9. Y. Li, Y.-S. Hu, H. Li, L. Chen, and X. Huang, J. Mater. Chem. A., 4, 96 (2016).

10. M. Dahbi, M. Kiso, K. Kubota, T. Horiba, T. Chafik, K. Hida, T. Matsuyama, and S. Komaba, J. Mater Chem. A., 5, 9917 (2017)

11. A. Ponrouch and M. R. Palacín, Electrochemistry Communications., 54, 51 (2015)

12. I. Breßler, J. Kohlbrecher, and A. F. Thünemann, Journal of Applied Crystallography., 48, $1587(2015)$

13. M. Wahid, Y. Gawli, D. Puthusseri, A. Kumar, M. V. Shelke, and S. Ogale, ACS Omega., 2, 3601 (2017).

14. E. Irisarri, A. Ponrouch, and M. R. Palacín, Journal of The Electrochemical Society., 162, A2476 (2015)

15. D. A. Stevens and J. R. Dahn, Journal of The Electrochemical Society., 147, 1271 (2000).

16. N. S. Babu, S. B. Tiwari, and R. Nageswara, Trends in Applied Sciences Research., 1, 204 (2006)

17. A. Cuesta, P. Dhamelincourt, A. Martinez-Alonso, J. Laureyns, and J.-M.-D. Tascon, Carbon., 38, 1523 (1994)

18. A.-C. Ferrari and J. Robertson, Physical Review B., 61, 14095 (2000).

19. J. Schwan, S. Ulrich, V. Batori, H. Ehrhardt, and S.-R.-P. Silva, J. Appl. Phys., 80, 440 (1996).

20. A. Sadezky, H. Muckenhuber, H. Grothe, R. Niessner, and U. Pöschl, Carbon., 43, 1731 (2005).

21. J. D. Herdman, B. C. Connelly, M. D. Smooke, M. B. Long, and J. H. Miller, Car bon., 49, 5298 (2011). 
22. K. S. W. Sing, D. H. Everett, R. A. W. Haul, L. Moscou, R. A. Pierotti, J. Rouquérol, and T. Siemieniewska, Pure \& Appl. Chem., 57, 603 (1985).

23. M. Thommes, K. Kaneko, A. V. Neimark, J. P. Olivier, F. Rodriguez-Reinoso, J. Rouquerol, and K. S. W. Sing, Pure and Applied Chemistry., 87 (2015).

24. J. C. Groen, L. A. A. Peffer, and J. Pérez-RamıF́rez, Microporous and Mesoporous Materials., 60, 1 (2003).

25. R. Kitaura, K. Seki, G. Akiyama, and S. Kitagawa, Angew. Chem. Int. Ed., 42, 428 (2003).

26. C. Matei Ghimbeu, J. Górka, V. Simone, L. Simonin, S. Martinet, and C. Vix-Guterl, Nano Energy., 44, 327 (2018).

27. F. Ehrburger-Dolle, M. Holz, and J. Lahaye, Pure \& Appl. Chem., 65, 2223 (1993).

28. A. P. Radlinski, M. Mastalerz, A. L. Hinde, M. Hainbuchner, H. Rauch, M. Baron, J. S. Lin, L. Fan, and P. Thiyagarajan, International Journal of Coal Geology, $\mathbf{5 9}$, 245 (2004).
29. D. Avnir and D. Farin, New J. Chem., 16, 439 (1992).

30. T. Rieker, S. Misono, and F. Ehrburger-Dolle, Langmuir., 15, 914 (1999).

31. C.-H. Sun, F. Li, Z. Ying, C. Liu, and H.-M. Cheng, Physical Review B., 69 (2004).

32. D. A. Stevens and J. R. Dahn, Journal of The Electrochemical Society., 148, A803 (2001).

33. V. Simone, A. Boulineau, A. de Geyer, D. Rouchon, L. Simonin, and S. Martinet, Journal of Energy Chemistry., 25, 761 (2016).

34. B. Zhang, C. M. Ghimbeu, C. Laberty, C. Vix-Guterl, and J.-M. Tarascon, Advanced Energy Materials., 6, 1501588 (2016).

35. Y. Bai, Z. Wang, C. Wu, R. Xu, F. Wu, Y. Liu, H. Li, Y. Li, J. Lu, and K. Amine, ACS Appl Mater Interfaces., 7, 5598 (2015).

36. M. Wahid, D. Puthusseri, Y. Gawli, N. Sharma, and S. Ogale, ChemSusChem., 11, 506 (2018). 